\title{
Article \\ Multi-Robot Leader Grouping Consistent Formation Control Method Research with Low Convergence Time Based on Nonholonomic Constraints
}

\author{
Hongchao Zhuang ${ }^{1, *(\mathbb{D}}$, Kailun Dong $\left.{ }^{1} \mathbb{(}\right)$, Ning Wang ${ }^{2}$ and Lei Dong ${ }^{1}$ \\ 1 School of Mechanical Engineering, Tianjin University of Technology and Education, Tianjin 300222, China; \\ dongkailun4869@163.com (K.D.); donglei_hit@163.com (L.D.) \\ 2 School of Information Technology Engineering, Tianjin University of Technology and Education, \\ Tianjin 300222, China; wangning811108@163.com \\ * Correspondence: zhuanghongchao_hit@163.com; Tel.: +86-022-88181083
}

check for updates

Citation: Zhuang, H.; Dong, K.; Wang, N.; Dong, L. Multi-Robot Leader Grouping Consistent Formation Control Method Research with Low Convergence Time Based on Nonholonomic Constraints. Appl. Sci. 2022, 12, 2300. https://doi.org/ 10.3390/app12052300

Academic Editors: Augusto Ferrante, Mihaiela Iliescu and Mingcong Deng

Received: 13 January 2022

Accepted: 20 February 2022

Published: 22 February 2022

Publisher's Note: MDPI stays neutral with regard to jurisdictional claims in published maps and institutional affiliations.

Copyright: (C) 2022 by the authors. Licensee MDPI, Basel, Switzerland. This article is an open access article distributed under the terms and conditions of the Creative Commons Attribution (CC BY) license (https:// creativecommons.org/licenses/by/ $4.0 /)$.

\begin{abstract}
Aiming at the formation and maintenance of the multiple formations of nonholonomic constrained multi-robots, a leader-follower formation control method under the grouping consistency is proposed on the trajectory tracking of a nonholonomic constrained mobile robots with the low convergence time. The distributed control structure in the leader-follower formation is adopted. The multi-robot cooperative formation is realized by using the consistency algorithm of graph theory. According to the graph theory, the communication topology matrixes are designed by the consistency algorithm. The mathematical model of nonholonomic constrained robot is established with the wheeled structure as the mobile structure under the nonholonomic constraints. Then the navigation following model is transformed into the error model of a local coordinate system through the global coordinate transformation. The formation control law of multi-robot cooperative motion is put forward based on the leader-follower model. Its convergence is proved by the Lyapunov function. By setting the reasonable communication protocol parameters, the MATLAB software (Natick, MA, USA, $\mathrm{R} 2016 \mathrm{~b}$ ) is employed on the simulation verification and result comparison. Through the comparison of the two leader formation control methods, the convergence time of the algorithm in this article can be $25 \%$ less than that of PFC. The effectiveness and feasibility of the formation control law are verified under the leader-follower method. The proposed control method lays a foundation for reducing the convergence time to improve the multi-robot cooperative motion under nonholonomic constraints.
\end{abstract}

Keywords: multi-robot; leader-follower formation; grouping consistency; convergence time; nonholonomic constraint

\section{Introduction}

In order to realize the common interests of some and even all individuals, the cluster activity is to unify the scattered individuals and social forces. They can adapt to different living conditions by living in groups. The purpose of cluster activities is to control, coordinate and restrict individuals and small groups living in groups. They manage the factors affecting the development of groups. The cluster activities ensure the survival and predation of groups to a certain extent. They can also coordinate with each other to maximize the interests of the group. At the same time, the cluster activities also greatly improve work efficiency.

Through the study and research of biological cluster activities, the swarm motion of bionic robots also develops. Usually, the cluster of robots is called a multi-robot system. The multi-robot system refers to a kind of complex system composed of multiple robots and their corresponding organization rules and information interaction protocols. It can complete specific tasks, such as multiple UAVs, multiple robots and so on. Among them, the organization rules determine the connection relationship between robots. The information 
interaction protocol is used to determine and update the state of robots. There are a large number of such examples in the real world [1-3], as shown in Figure 1. At the same time, the multi-robot cluster movement also began to develop. The cluster movement can not only improve the energy utilization, but also improve the efficiency of movement. There are also good solutions for the large-scale task requirements.

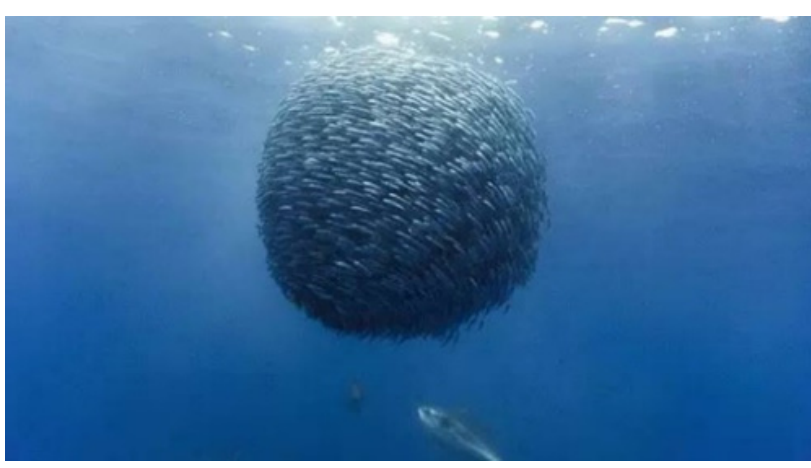

(a)

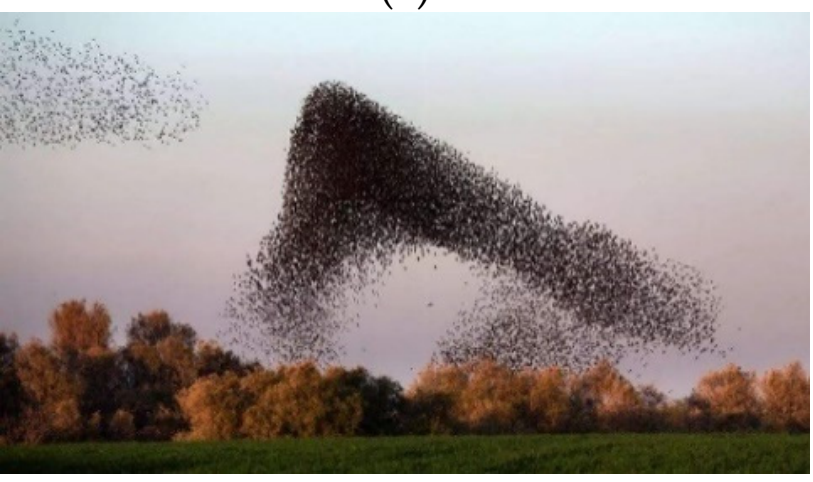

(c)

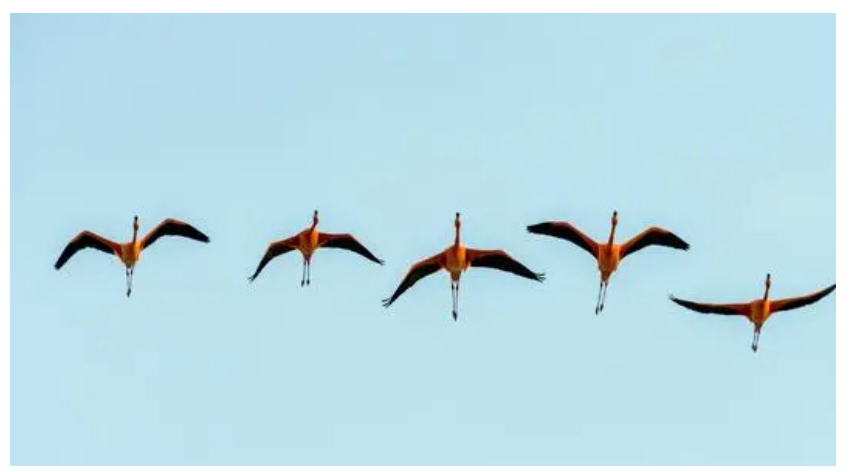

(b)

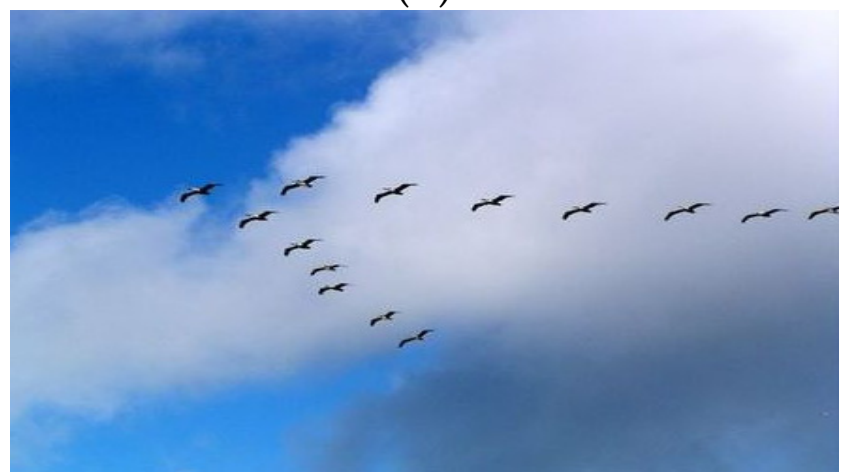

(d)

Figure 1. Example of multi-robot cooperation in nature: (a) defense strategy of fish school; (b) migration of flamingo; (c) when sandpipers are frightened; (d) migration of geese.

After more than 20 years of development, the multi-robot collaboration has shown a wide range of applications in the military, national defense, industry, life and other fields [4]. For example, it has shown great advantages in specific applications such as the formation patrol, environmental exploration, assembly-line production and warehousing and transportation [5-7]. Some specific application tasks include multi-robot cooperative map exploration, large object transportation, target capture and so on. They require the robots in the team to form a specific formation structure. According to the environmental constraints and the different task requirements, they can keep the formation unchanged or change the formation shape in the process of task execution. The common formations include the straight line, wedge, column, triangle, double row, etc. [3], as shown in Figure 2. Arrows between robots indicate the flow of information. The arrow in front of the robot $\mathrm{R}_{1}$ indicates the direction of motion of the multi-robot system. The research work of this article will take the mobile robot as the main research object.

Therefore, the research boom of multi-robot formation control has been triggered. It has become one of the key technologies in the field of multi-robot cooperation and coordination. Taking the nonholonomic multi-mobile robot as the research object and the cooperative formation as the application background, the key technologies of multi-mobile robot motion formation control is studied in this article.

Motion control is one of the important research topics in the multi-robot systems. By establishing effective communication control strategies among robots, the multi-robot 
systems can cooperate to complete a complex task. The multi-robot systems realize the expected formation. At present, the commonly used robot formation control algorithms include the virtual structure method, based behavior method, artificial potential field method, leader-follower method [8-11]. The advantage of the leader-follower method is that the formation control structure is simple and easy to implement. In the formation, only the desired path or other behavior of the leader needs to be set, and then the follower can follow the leader with a predetermined position offset to realize the formation control. For this reason, the leader-follower method is widely used in practical engineering. Hence, the leader-follower method can be employed for the formation control.

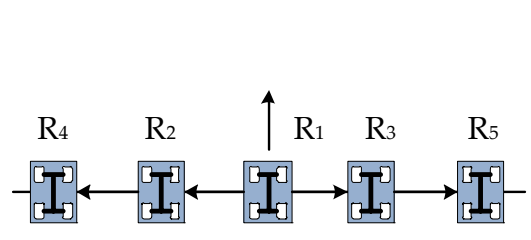

(a)

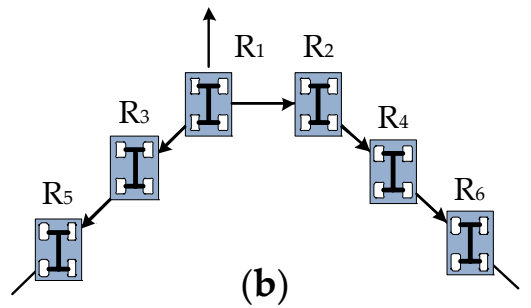

(b)

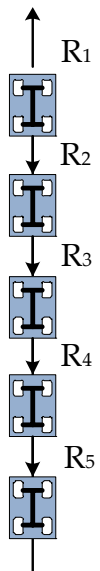

(c)

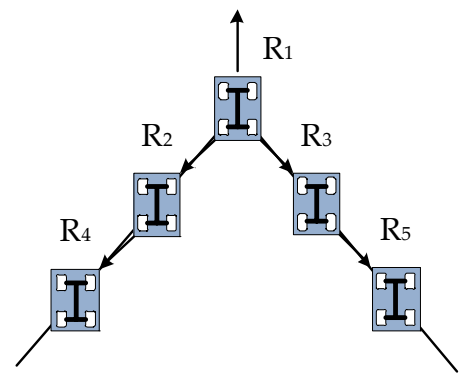

(d)

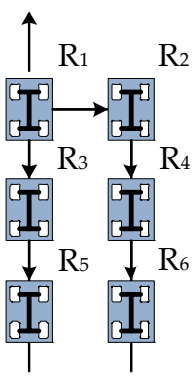

(e)

Figure 2. Common multi-robot formation [3]: (a) straight line; (b) wedge; (c) column; (d) triangle; (e) double row.

The concept of leader-follower was first proposed and successfully applied to the formation control of mobile robots $[12,13]$. As the most commonly used formation control method at present, its basic idea is that all formation members are designated as the leader or follower. The leader controls the movement trend of the whole formation by navigating along the predetermined or temporarily set path. According to the distance and azimuth information relative to the leader, the follower follows the leader to realize formation control. The advantage of the leader-follower method is that the formation control structure is simple and easy to implement. In the formation, only the desired path or other behavior of the leader needs to be set, and then the follower can follow the leader with a predetermined position offset to realize the formation control. For the above reasons, the leader-follower method is widely used in practical engineering. The disadvantage of the method is that the formation system depends too much on the leader.

A new multi-robot formation control method is known as the leader-follower method. The stability of the algorithm was proved by the simulation results [14]. The formation control problem was transformed into the problem of state consistency by the transformation of variables. A controller was designed to make a group of nonholonomic robots gradually converge to the desired formation along the specified trajectory [15]. When the external input was limited, the navigation and following formation problem of nonholonomic multi-mobile robots was discussed. Under the nonholonomic constraints, the external distributed sensor method was applied. The finite-time convergence problem of 
multi-robot cooperative control was studied $[16,17]$. The motion consistency problem of complex multi-robots was studied, and the dynamic formation of multi-mobile robots was realized by designing a distributed formation control law $[18,19]$. The time-varying stability problem of planar multi-robots with nonholonomic constraints was studied [20,21]. Assuming that there were many external disturbances in the system. The finite-time containment control problem of second-order multi-robot systems with mismatched disturbances was studied $[21,22]$. The adaptive tracking control of omnidirectional mobile robots was analyzed under the conditions of state constraints and input saturation [23]. Assuming that there were multiple leaders, the cluster containment control problem of the robot system was researched [24]. The acquisition spacecraft operation of the space robot was analyzed. The collision avoidance compliance compound active disturbance rejection control problem of a robot was studied. Considering the post-capture unstable combined system, collision avoidance and compliant composite error active disturbance rejection control is proposed for the stabilization control. Numerical simulation verifies the effectiveness of the proposed collision avoidance and compliant control method [25,26].

Most of the above studies focus on the leader-follower method implementation and external distributed sensor fusion. The multi-robot cooperative formation needs to closely combine the theories and algorithm simulation. This article combines the nonholonomic constrained mobile robot, graph theory, leader-follow formation control and trajectory tracking. The error analysis of the nonholonomic multi-robot system is implemented. Based on information consistency, the formation realization of single formation and multicombination formation is completed. Through the existing research results, in Section 2, the communication topology matrixes are designed by using a consistency algorithm based on the graph theory. The formation control law of multi-robot cooperative motion based on the leader-follow model is constructed. In Section 3, the mathematical model of a wheeled mobile robot under the nonholonomic constraints is established. Then, the leader-follower model is transformed into the local coordinate system error model through the global coordinate transformation. The Lyapunov function is used for the theoretical analysis. In Section 4, by setting the reasonable communication protocol parameters, the simulation results are competed with another algorithm of leader-follower formation by using MATLAB software. Based on the leader-follower method, the effectiveness and feasibility of the formation control law are verified by compared to other formation control strategies. In the final section, the conclusions are presented.

\section{Multi-Robot Cooperative Formation Control Based on Group Formation}

\subsection{Graph Theory Description}

The control cooperative multi-robot dynamic system is a dynamic problem related to each other through a communication diagram, which shows the information flow between each node. The goal of cooperative control is to design control protocols for each node and to ensure the state synchronization behavior of all nodes in a specific sense. In a cooperative system, any control protocol must carry out the distributed control according to its specified graph topology. The control protocol of each node is only allowed to rely on the information about the node and its neighbor nodes in the graph. The communication restrictions imposed by the topology may seriously limit the work. This work is completed by the local distributed control protocol on each node.

In multi-robot cooperative formation, this article focuses on the behavior and interaction of dynamic systems. These dynamic systems are interconnected through the link of a communication network. The communication network is modeled as a graph with directed edges corresponding to the allowed information flow between systems. Each robot in the formation is modeled as a node in the graph. The most basic problem is how the graph topology interacts with the local feedback control protocol of nodes to generate the overall behavior of interconnected nodes. This problem can be solved by the graph theory. The following shows some basic graph theory concepts that are essential in the study of multi-robot cooperative formation. 
The topological graph can be represented as $G=(V, E)$, where $V=\left\{v_{1}, \ldots, v_{N}\right\}$ represents the set of $\boldsymbol{N}$ nodes, and $\boldsymbol{E}$ represents the set of $\boldsymbol{E}$ edges. Assuming that the graph is a simple directed graph, that is $\left(v_{i}, v_{j}\right) \notin \boldsymbol{E}$. At this time, there are no multiple edges between the same pair of nodes. The elements $\left(v_{i}, v_{j}\right)$ in the set $E$ represent the edges from node $v_{j}$ to node $v_{i}$. In the formation, it represents that the information flows from node $v_{j}$ robot to node $v_{i}$ robot. The out degree of node $v_{i}$ robot $d_{i}^{o}$ represents the number of edges with it as the data sending end. The in degree $d_{i}$ represents the number of edges with it as the data receiving end.

The structure and properties of graphs can be studied by studying the properties of some matrices related to graphs, which is algebraic graph theory. After the weight $a_{i j}$ of the edge is given, the graph can be represented by an adjacency matrix or connected matrix $\boldsymbol{A}=\left(a_{i j}\right)$, where when $\left(v_{i}, v_{j}\right) \notin \boldsymbol{E}, a_{i j}=1$, otherwise $a_{i j}=0$. The weighted penetration of point $i$ at this time can be expressed as the sum of all values in row $i$ of matrix $A$, as shown in Equation (1). Then

$$
d_{i}=\sum_{j=1}^{N} a_{i j}
$$

Then, the weighted output can be expressed as Equation (2). Then

$$
d_{i}^{o}=\sum_{j=1}^{N} a_{j i} .
$$

Both in degree and out degree are local properties of the graph. In formation topology communication, the out degree can reflect the impact of the node on other robots in the formation, that is, the greater the out degree, the greater the impact of the node robot on the formation consistency.

The diagonal penetration matrix and graph Laplacian matrix are defined as $\boldsymbol{D}=\operatorname{diag} d_{i}$ and $\boldsymbol{L}=\boldsymbol{D}-\boldsymbol{A}$, respectively. Many properties of a graph can be studied as the properties of graph Laplacian matrix. It is of great significance in the study of graph theory of dynamic multi-node systems. In order to explain the relationship between the directed graph and adjacency matrix, between the diagonal matrix and Laplace matrix, a directed graph of a multi-robot system is assumed as shown in Figure 3. So, its adjoint matrix $A$, diagonal matrix $\boldsymbol{D}$ and Laplacian matrix $L$ can be shown in Equation (3).

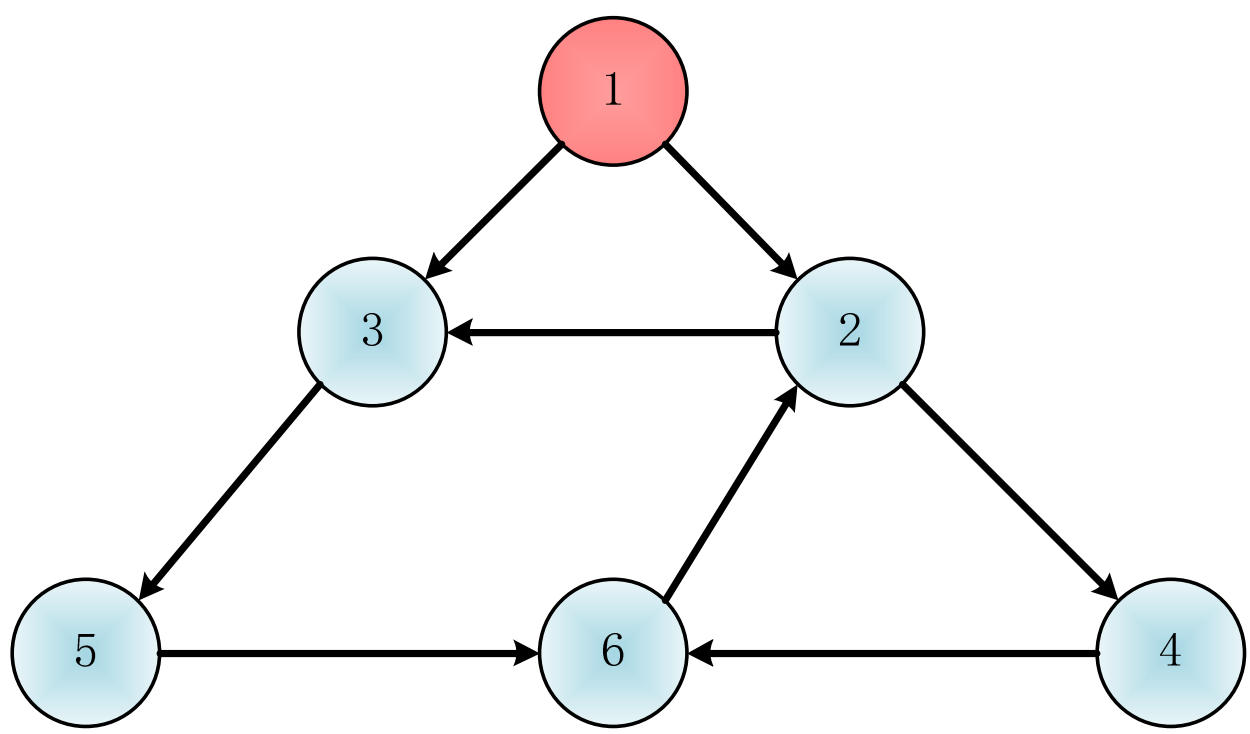

Figure 3. Directed graph of multi-robot system.

In Figure 3, the red circle represents the leader robot. The blue circle represents the following robot. The number represents the serial number of the robot. The arrow 
represents the directed edge. The corresponding matrixes of the directed graph are shown as follows in Equation (3). Then

$$
\boldsymbol{A}=\left(\begin{array}{llllll}
0 & 0 & 0 & 0 & 0 & 0 \\
1 & 0 & 0 & 0 & 0 & 1 \\
1 & 1 & 0 & 0 & 0 & 0 \\
0 & 1 & 0 & 0 & 0 & 0 \\
0 & 0 & 1 & 0 & 0 & 0 \\
0 & 0 & 0 & 1 & 1 & 0
\end{array}\right), \boldsymbol{D}=\left(\begin{array}{llllll}
0 & 0 & 0 & 0 & 0 & 0 \\
0 & 2 & 0 & 0 & 0 & 0 \\
0 & 0 & 2 & 0 & 0 & 0 \\
0 & 0 & 0 & 1 & 0 & 0 \\
0 & 0 & 0 & 0 & 1 & 0 \\
0 & 0 & 0 & 0 & 0 & 2
\end{array}\right), \boldsymbol{L}=\left(\begin{array}{cccccc}
0 & 0 & 0 & 0 & 0 & 0 \\
-1 & 2 & 0 & 0 & 0 & -1 \\
-1 & -1 & 2 & 0 & 0 & 0 \\
0 & -1 & 0 & 1 & 0 & 0 \\
0 & 0 & -1 & 0 & 1 & 0 \\
0 & 0 & 0 & -1 & 0 & 2
\end{array}\right),
$$

The characteristic structure of the graph Laplacian matrix $L$ plays a key role in the dynamic system analysis on the graph. The eigenvalue of $L$ is analyzed below, and the approximate form of the graph Laplacian matrix is defined as follows in Equation (4).

Then

$$
L=M J M^{-1},
$$

In Equation (4), the Jordan matrix $J$ and the transformation matrix $\boldsymbol{M}$ are shown as Equation (5). Then

$$
\left\{\begin{array}{l}
\boldsymbol{M}=\left(\begin{array}{cccc}
V_{1} & V_{2} & \cdots & V_{N}
\end{array}\right) \\
\boldsymbol{J}=\left(\begin{array}{cccc}
\lambda_{1} & 1 & & \\
& \lambda_{2} & 1 & \\
& & \cdots & 1 \\
& & & \lambda_{N}
\end{array}\right),
\end{array}\right.
$$

In Equation (5), the eigenvalue $\lambda_{i}$ and the right eigenvectors $r_{i}$ of $\boldsymbol{M}$ satisfy Equation (6). Then

$$
\left(\lambda_{i} \boldsymbol{I}-\boldsymbol{L}\right) r_{i}=0,
$$

where $I$ is the identity matrix. The left eigenvector $\omega_{i}$ of matrix $M$ satisfies Equation (7).

Then

$$
\left(\begin{array}{cc}
\omega_{i} & 0 \\
0 & \omega_{i}^{T}
\end{array}\right)\left(\begin{array}{c}
\lambda_{i} \boldsymbol{I}-\boldsymbol{L} \\
r_{i}
\end{array}\right)=\left(\begin{array}{l}
0 \\
1
\end{array}\right) .
$$

where $\omega_{i}^{T}$ is the transpose vector of the eigenvector $\omega_{i}$.

Since the sum of all row elements of matrix $L$ is 0 , if and only if $L$ has a unique eigenvector $c$ such that $L c=0$ and its eigenvalue is 0 . The rank of $L$ is $(N-1)$. Graph $G$ has a spanning tree, which plays an important role in forming the stability of formation.

\subsection{Formation Control Principle}

The distributed control structure in the leader-follower formation is adopted in this article. The formation control is realized by using the consistency algorithm given below on the basis of the previous graph theory. The consistency is defined as one or some states of all robots in a multi-robot system that tend to be consistent with the evolution of time. The leader-follower formation is a classical formation form. The main idea of the first-order formation algorithm based on consistency is to control the relative position and speed between the leader and the follower. When these quantities reach a certain stable state, the formation is realized, in which the leader robot plays a vital role. The first-order continuous system model of the formation system is Equation (8) as follows.

Then

$$
\dot{x}_{i}=u_{i},
$$

where $x_{i}$ and $u_{i}$ represent the state quantity and input quantity of node $i$ respectively, and $x_{i}, u_{i} \in \boldsymbol{R}_{n}, n$ represent the dimension of state quantity. Ideally, consider the following forms of control input. Then

$$
u_{i}=\sum_{j \in N_{i}} a_{i j}\left(x_{j}-x_{i}\right),
$$


where $a_{i j}$ is the formation adjacency matrix element and $N_{i}$ is the neighbor set of member $i$. By substituting Equation (9) into Equation (8) and introducing the global state vector $\mathbf{x}=\left(x_{1}, \ldots, x_{n}\right)^{\mathrm{T}} \in \boldsymbol{R}_{n}$, the global dynamic relationship can be obtained as follows in Equation (10). Then

$$
\dot{x}=-D x+A x=-L x,
$$

Based on Equation (10), it can be concluded that the closed-loop dynamic characteristics of the formation depend on the matrix $L$. In addition, if and only if the topological graph $G$ of the formation has a spanning tree, $-L$ eigenvalues are located in the left half plane of the complex plane. Hence, Equation (10) can ensure the consistency of the system. The final state value is shown as follows.

Then

$$
c=\sum_{i=1}^{N} p_{i} x_{i}(0),
$$

In discrete time, Equation (8) can be reduced to Equation (12). Then

$$
x_{i}(k+1)=x_{i}(k)+u_{i}(k),
$$

In this subject, it is assumed that a scene is a scene in which the leader leads other robots to complete a certain distance and move from the starting point to a target point. Ideally, the algorithm of the follower in the formation adopts the following form. Then

$$
u_{i}(k)=\varepsilon \sum_{j \in N_{i}} a_{i j}\left(x_{j}(k)-x_{i}(k)-r_{i j}(k)\right),
$$

Among $\varepsilon>0, r_{i j}$ represents the relative position between robot $i$ and robot $j$, which is related to the distance between them, while the control algorithm of leader $N$ is Equation (14). Then

$$
u_{N}(k)=m+k \boldsymbol{D}(k)+\sum_{i \in N_{i}} a_{N i} r_{N i}(k) .
$$

where $r_{N j}$ represents the relative position between the leader and other robots, $m$ and $k$ are constants, $\boldsymbol{D}(k)$ represents the distance from the leader to the target point at time $k$, and the speed of the leader is affected by the adjacent nodes and the distance from the leader to the target point.

\subsection{Implementation of Group Formation Control Algorithm Based on Consistency for Nonholonomic Systems}

The basic idea of the consistency algorithm is that each robot uses the robot network to transmit information. It designs an appropriate distributed control algorithm. It couples the robot dynamics with the robot communication topology to form a complex system, so as to realize the continuous or synchronization of the state.

The goal of this section is to design a unified grouping consistent formation (GCF) scheme for a system that can be divided into $k$ groups and composed of $N$ incomplete mobile multi-robots to meet the large-scale multi-robot system formation. $\boldsymbol{V}=\left\{v_{1}, \ldots, v_{N}\right\}$ represents a collection of $N$ nodes, $V^{k}$ represents the $k$-th group, and $V^{k} \in V$, this article adopts the multi-group formation mode to realize a large-scale multi-mobile robot system, as shown in the following figure. $\mathrm{R}_{k}{ }^{L}$ represents the leading robot of the $k$-th group, and $\mathrm{R}_{k-s}$ represents the $s$-th follower robot of the $k$-th group, as shown in Figure 4 . While the leader robot $\mathrm{R}_{k}{ }^{L}$ forms a formation with the inside of the group, it also forms a formation with the leader robots of other groups. The leader robot only has a communication topology diagram with the robots in the group and the leader robots in each group. The group formation greatly increases the size of the formation, and also reduces the amount of information processing of the navigator in the multi-robot system. It is of great significance to a large number of robot systems. 


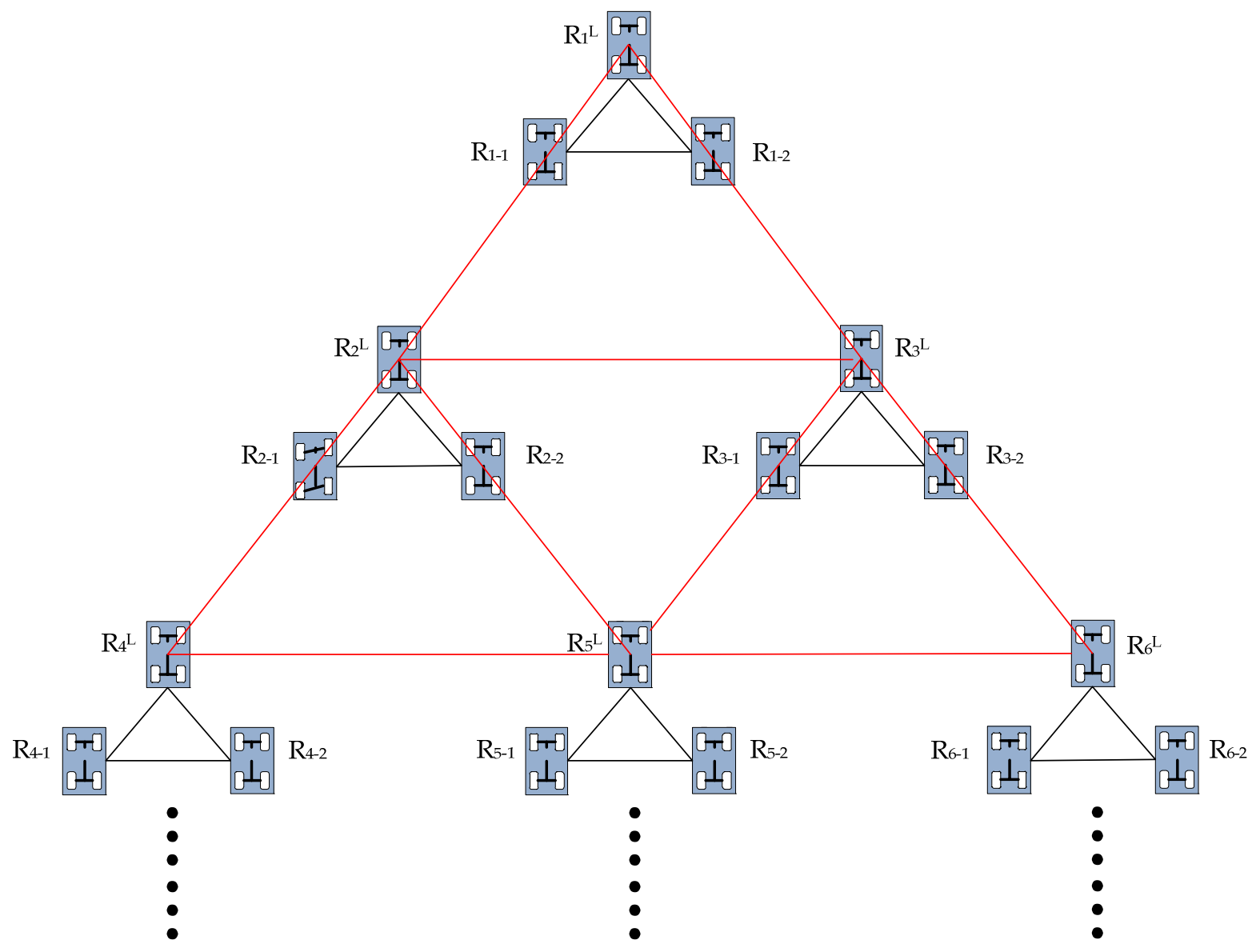

Figure 4. Communication topology of grouping consistent formation.

The corresponding communication topology matrix is shown as follows in Equation (15). Then

$$
\left\{\begin{array}{l}
\boldsymbol{A}_{k}=\left\{a_{k-i j}\right\} \\
\boldsymbol{A}^{m \times m}=\left\{\boldsymbol{A}_{k}\right\}
\end{array},\right.
$$

In order to verify the formation consistency algorithm, five robots are used to form an experimental robot formation in the form of leader-follower to find a stable communication topology matrix $A^{\prime}$ as shown in Equation (16). Then

$$
A^{\prime}=\left(\begin{array}{lllll}
0 & 1 & 1 & 1 & 1 \\
0 & 0 & 0 & 0 & 1 \\
0 & 0 & 0 & 1 & 1 \\
0 & 0 & 1 & 0 & 1 \\
0 & 0 & 0 & 0 & 0
\end{array}\right),
$$

After using the communication topology matrix to make a simulation experiment in Section 4, the formation and its communication topology are shown in Figure 5. The arrow indicates the direction of information flow. In Figure 5, it can be seen that the outgoing degree of node 5 is the largest. So, node 5 is the key node. That is the leader robot. The outgoing degree of node 1 is 0 , which has the least impact on the formation. Both in and out degrees are local properties of the graph. In formation topology communication, their out and in degrees can reflect the influence of nodes on other robots in the formation. The larger the exit degree, the greater the influence of the node robot on the formation consistency. When the penetration is larger, the influence of the node robot on the formation consistency is smaller. 


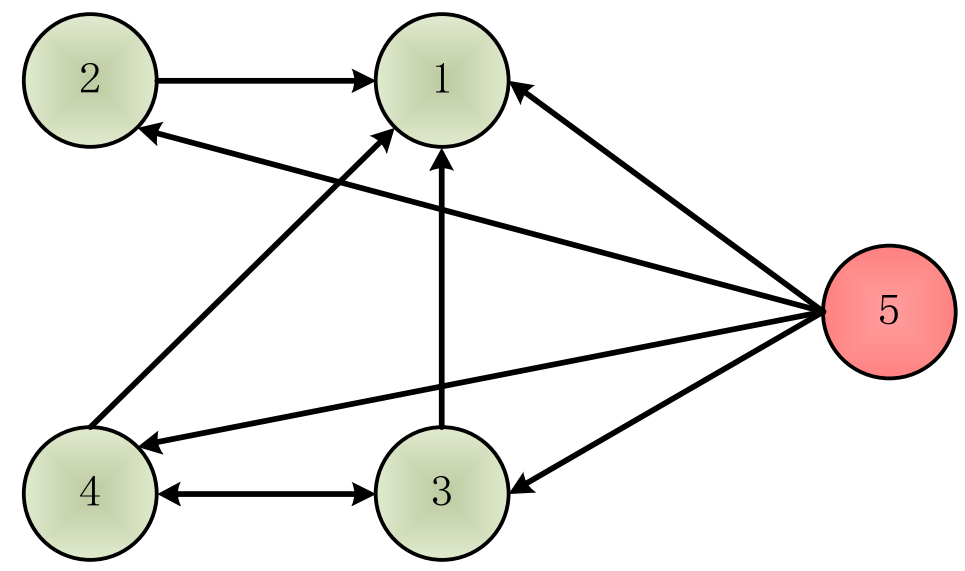

Figure 5. The formation and communication topology of the topology matrix $A^{\prime}$.

\section{Mathematical Model of Formation Operation Control}

The basic idea of the leader-follower method is to select a leader of robots and set its trajectory. The following robot tracks the trajectory of the leader robot in real-time. The trajectory of the whole formation is determined by the leader robot. The formation shape is maintained by the relative position relationship between the tracking robot and the leader. In the motion process of a multi-robot system based on the leader-follower model, in order to avoid collision between robots during tracking, a virtual robot generated by the leader robot and completely obtains the state information of the leader robot is introduced to calculate the state error between the follower robot and the virtual robot and make it converge to zero. Based on the above analysis, the formation problem of multi-robot can be transformed into a trajectory tracking problem of the following robot to leader robot.

\subsection{Robot System Model}

The nonholonomic constrained robots are mainly wheeled mobile structures, including wheeled, wheel leg composite, crawler and so on. In order to facilitate the theoretical analysis of the algorithm, this article takes the mathematical model of the four-wheel mobile robot as an example, as shown in Figure 6. The front and rear wheelbase of the robot is $L$. The distance between the midpoint of the wheel and the midpoint of the robot system is $d$, as shown in Equation (17). The motion direction angle of the robot is $\theta$. It is assumed that the linear velocity and angular velocity of the robot motion are $v$ and $\omega$. The linear speed of the wheel is $u$. Then

$$
\left\{\begin{array}{l}
d=L \cos \varphi \\
\omega=(u \cos \varphi) / d \\
v=u \sin \varphi
\end{array}\right.
$$

The influence of sliding can be ignored in this article, so the mobile robot belongs to a pure rolling sliding free robot. For the robot with pure rolling and no sliding, it is necessary to meet the nonholonomic constraints, as shown in Equation (18). Then

$$
\left(\begin{array}{ll}
\dot{x} & \dot{y}
\end{array}\right)\left(\begin{array}{cc}
\sin \theta & \cos \theta \\
-\cos \theta & \sin \theta
\end{array}\right)=\left(\begin{array}{ll}
d \omega & 0
\end{array}\right),
$$

By expressing all state quantities of the mobile robot, its mathematical model can be obtained, as shown in Equation (19). Then

$$
\left(\begin{array}{l}
\dot{x} \\
\dot{y} \\
\dot{\theta}
\end{array}\right)=\left(\begin{array}{cc}
\cos \theta & d \sin \theta \\
\sin \theta & -d \cos \theta \\
0 & 1
\end{array}\right)\left(\begin{array}{c}
v \\
\omega
\end{array}\right),
$$


where $v$ and $w$ are the parameters that can be directly controlled, $x, y$ and $\theta$ are the state quantities of the system. The number of parameters that can be controlled is less than the number of state qua tities of the system. Therefore, the system is an underactuated system, also known as a nonholonomic constraint system.

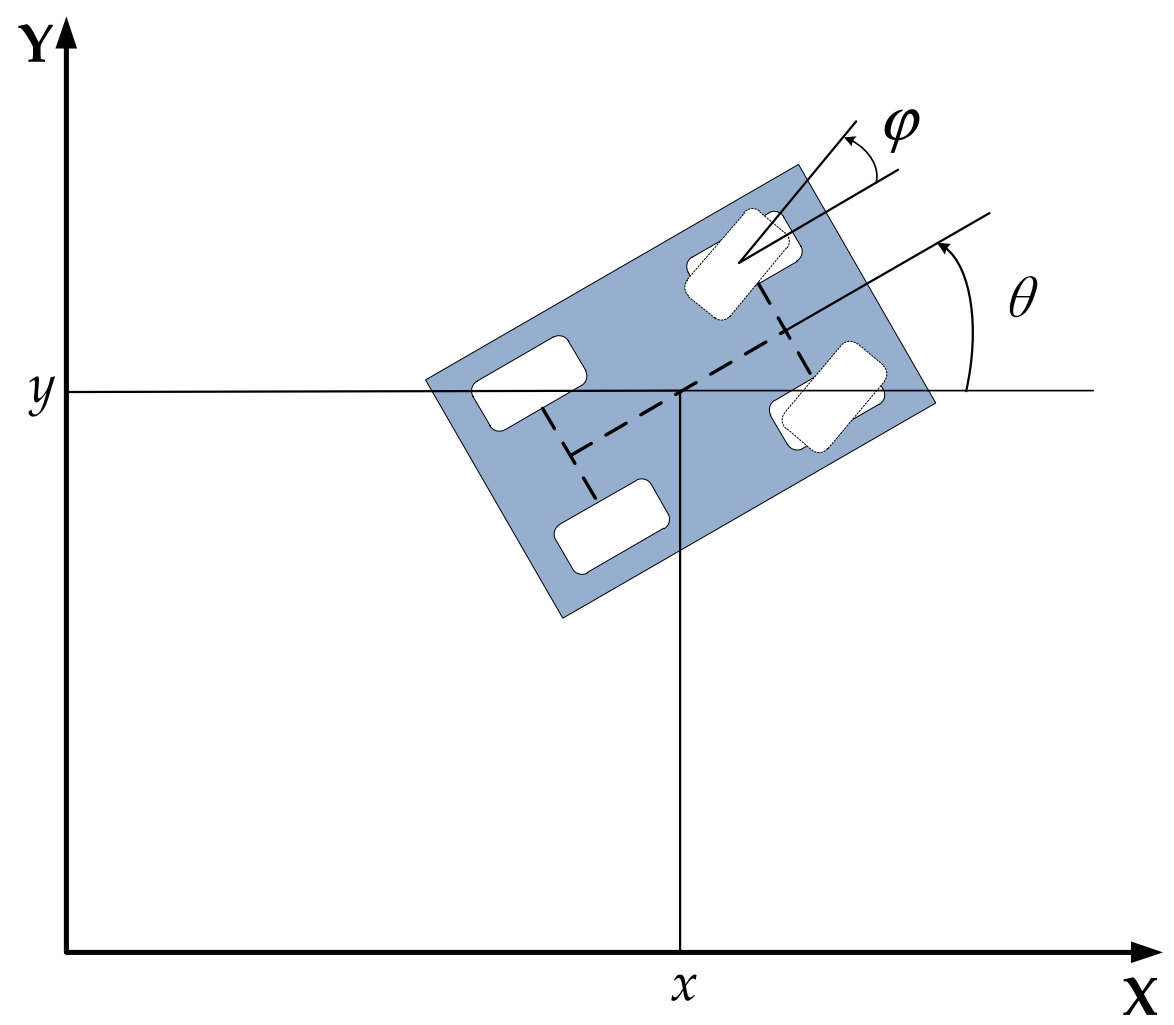

Figure 6. Mathematical model of wheeled mobile robot.

\subsection{Mathematical Model of Leader}

According to the conditions to be met by the robot model, Section 3.2 gives the state quantities of the leader robot as $x_{L}, y_{L}$ and $\theta_{L}$. Then the ideal state quantities of the follower robot can be expressed as Equation (20).

Then

$$
\left(\begin{array}{c}
x_{i}^{d}(t) \\
y_{i}^{d}(t) \\
\theta_{i}^{d}
\end{array}\right)=\left(\begin{array}{ccc}
x_{L}(t) & -\cos \left(\theta_{L}(t)+\varphi_{i L}^{d}\right) & 0 \\
y_{L}(t) & -\sin \left(\theta_{L}(t)+\varphi_{i L}^{d}\right) & 0 \\
0 & 0 & 1
\end{array}\right)\left(\begin{array}{c}
1 \\
l_{i L}^{d} \\
\theta_{L}
\end{array}\right),
$$

where the ideal state quantities of the follower robot are as $x_{i}^{d}, y_{i}^{d}$ and $\theta_{i}^{d} \cdot \varphi_{i L}^{d}$ is the angle between the ideal state of the follower robot and the leader robot in the coordinate system of the leader robot. $l_{i L}^{d}$ is the distance between the ideal state of the follower robot and the leader robot.

The leader formation control algorithm can be introduced virtual leader robots as expectation analysis. In a nonholonomic system, both the virtual leader robot and the navigation robot meet the nonholonomic constraints. The mathematical model of the virtual leader robot can be described as Equation (21). Then

$$
\left(\begin{array}{l}
\dot{x}_{v} \\
\dot{y}_{v} \\
\dot{\theta}_{v}
\end{array}\right)=\left(\begin{array}{cc}
\cos \theta_{v} & d \sin \theta_{v} \\
\sin \theta_{v} & -d \cos \theta_{v} \\
0 & 1
\end{array}\right)\left(\begin{array}{c}
v_{v} \\
\omega_{v}
\end{array}\right),
$$


where $v_{v}$ and $\omega_{v}$ are the parameter of the virtual leader robot. $x_{v}, y_{v}$ and $\theta_{v}$ are the state quantities of the virtual leader robot. $\dot{x}_{v}, \dot{y}_{v}$ and $\dot{\theta}_{v}$ are the first derivatives of $x_{v}, y_{v}$ and $\theta_{v}$ respectively.

According to Equation (19), the mathematical model of the following robot is shown in Equation (22). Then

$$
\left(\begin{array}{c}
\dot{x}_{f} \\
\dot{y}_{f} \\
\dot{\theta}_{f}
\end{array}\right)=\left(\begin{array}{cc}
\cos \theta_{f} & d \sin \theta_{f} \\
\sin \theta_{f} & -d \cos \theta_{f} \\
0 & 1
\end{array}\right)\left(\begin{array}{c}
v_{f} \\
\omega_{f}
\end{array}\right),
$$

where $v_{f}$ and $\omega_{f}$ are the parameter of the virtual leader robot. $x_{f}, y_{f}$ and $\theta_{f}$ are the state quantities of the virtual leader robot. $\dot{x}_{f}, \dot{y}_{f}$ and $\dot{\theta}_{f}$ are the first derivatives of $x_{f}, y_{f}$ and $\theta_{f}$, respectively.

\subsection{Leader-Follower System Model}

The simple diagram of the leader-follower system model is shown in Figure 7. According to Equations (20)-(22), the state error between leader formation multi-robot systems in this article is Equation (23).

Then

$$
\left(\begin{array}{c}
x_{i e} \\
y_{i e} \\
\theta_{i e}
\end{array}\right)=\left(\begin{array}{ccc}
\cos \theta_{i} & \sin \theta_{i} & 0 \\
-\sin \theta_{i} & \cos \theta_{i} & 0 \\
0 & 0 & 1
\end{array}\right)\left(\begin{array}{c}
x_{i}^{d}-x_{i} \\
y_{i}^{d}-y_{i} \\
\theta_{i}^{d}-\theta_{i}
\end{array}\right),
$$

where $x_{i e}, y_{i e}$ and $\theta_{i e}$ are the state errors of the system. The ideal state quantities of the follower robot are defined as $x_{i}^{d}, y_{i}^{d}$ and $\theta_{i}^{d} . x_{i}, y_{i}$ and $\theta_{i}$ are the state quantities of the $i$-th robot.

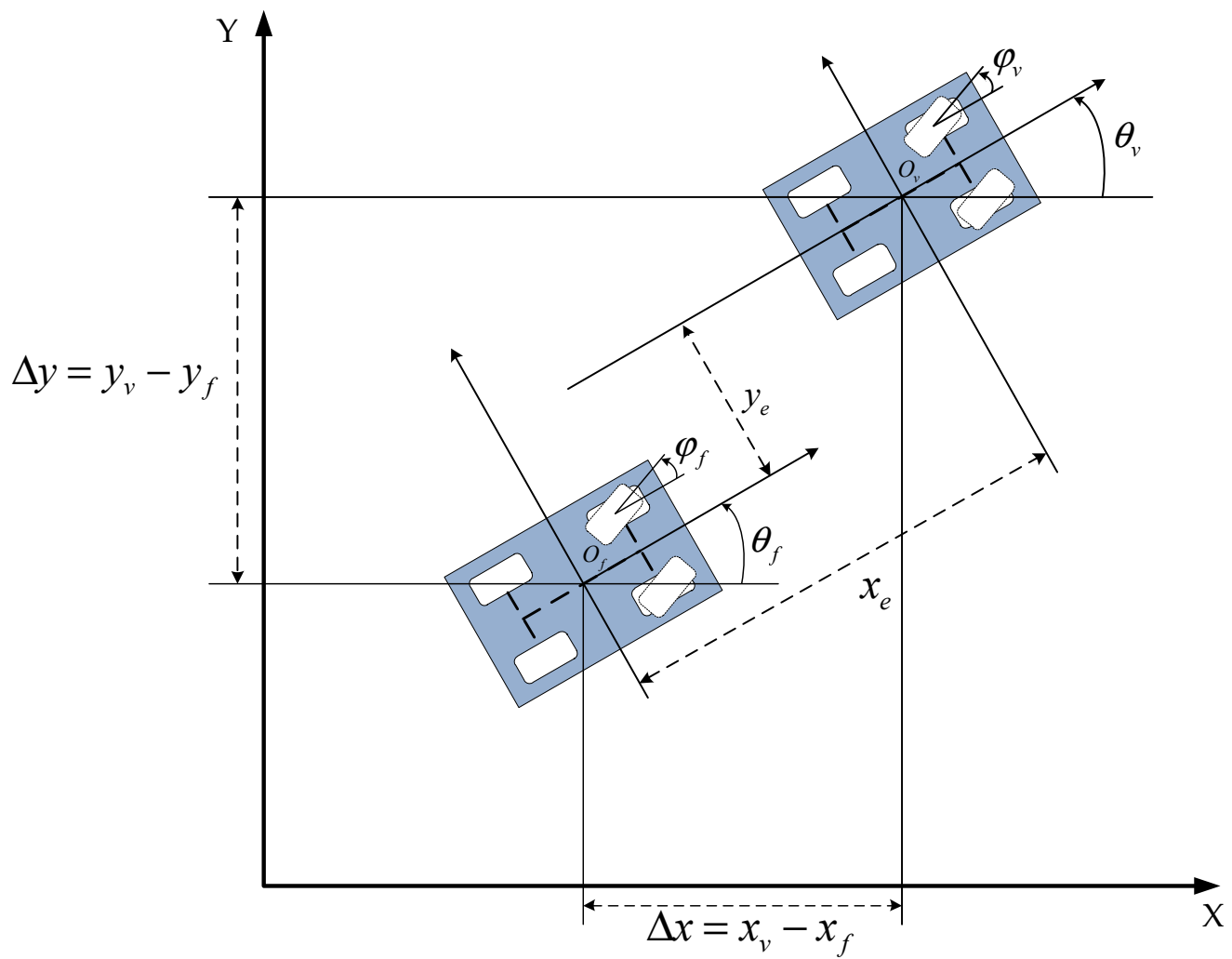

Figure 7. Simple diagram of leader-follower system model. 
The virtual robot proposed in the previous section is used as error analysis. Equation (24) can be obtained from the coordinate relative position relationship between the virtual leader robot and the following robot. Then

$$
\left(\begin{array}{l}
x_{i e} \\
y_{i e} \\
\theta_{i e}
\end{array}\right)=\left(\begin{array}{ccc}
\cos \theta_{f} & \sin \theta_{f} & 0 \\
-\sin \theta_{f} & \cos \theta_{f} & 0 \\
0 & 0 & 1
\end{array}\right)\left(\begin{array}{l}
x_{v}-x_{f} \\
y_{v}-y_{f} \\
\theta_{v}-\theta_{f}
\end{array}\right),
$$

Sorting out the state error, deriving Equation (24), and substituting Equations (21) and (20), the following Equation (25) can be obtained. Then

$$
\left\{\begin{aligned}
\dot{x}_{i e}= & \omega_{f} y_{i e}+\cos \theta_{f}\left(v_{v} \cos \theta_{v}+d \omega_{v} \sin \theta_{v}\right)-\cos \theta_{f}\left(v_{f} \cos \theta_{f}+d \omega_{f} \sin \theta_{f}\right) \\
& +\sin \theta_{f}\left(v_{v} \sin \theta_{v}-d \omega_{v} \cos \theta_{v}\right)-\sin \theta_{f}\left(v_{f} \sin \theta_{f}-d \omega_{f} \cos \theta_{f}\right) \\
= & \omega_{f} y_{i e}+v_{v} \cos \theta_{i e}+\omega_{v} d \sin \theta_{i e}-v_{f} \\
\dot{y}_{i e}= & -\omega_{f} x_{i e}-\sin \theta_{f}\left(v_{v} \cos \theta_{v}+d \omega_{v} \sin \theta_{v}\right)-\sin \theta_{f}\left(v_{f} \cos \theta_{f}+d \omega_{f} \sin \theta_{f}\right) \\
& +\cos \theta_{f}\left(v_{v} \sin \theta_{v}-d \omega_{v} \cos \theta_{v}\right)-\cos \theta_{f}\left(v_{f} \sin \theta_{f}-d \omega_{f} \cos \theta_{f}\right) \\
= & -\omega_{f} x_{i e}+v_{v} \sin \theta_{i e}+\omega_{f} d-\omega_{v} d \cos \theta_{i e} \\
\dot{\theta}= & \omega_{v}-\omega_{f}
\end{aligned}\right.
$$

The state error after coordinate transformation can be described as Equation (26). Then

$$
\left(\begin{array}{c}
\dot{x}_{i e} \\
\dot{y}_{i e} \\
\dot{\theta}_{i e}
\end{array}\right)=\left(\begin{array}{cccc}
\cos \theta_{i e} & -1 & d \sin \theta_{i e} & y_{i e} \\
\sin \theta_{i e} & 0 & -d \cos \theta_{i e} & d-x_{i e} \\
0 & 0 & 1 & -1
\end{array}\right)\left(\begin{array}{c}
v_{v} \\
v_{f} \\
\omega_{v} \\
\omega_{f}
\end{array}\right),
$$

The input of the following robot $\boldsymbol{R}_{f}$ are $v$ and $\omega . v$ and $\omega$ can be adjusted to satisfy the following Equation (27). Then

$$
\left\{\begin{array}{l}
\lim _{t \rightarrow t_{0}}\left|x_{i e}\right|=0 \\
\lim _{t \rightarrow t_{0}}\left|y_{i e}\right|=0 \\
\lim _{t \rightarrow t_{0}}\left|\theta_{i e}\right|=0
\end{array}\right.
$$

In order to make the tracking error converge to zero, the multi-robot formation control law based on the leader-follower method can be designed as Equation (28). Then

$$
\left\{\begin{array}{l}
v_{f}=v_{v} \cos \theta_{i e}+k_{x}\left(x_{i e}-d\left(1-\cos \theta_{i e}\right)\right)-k_{\theta} \theta_{i e} \omega_{f} \\
\omega_{f}=\omega_{v}+v_{v}\left(k_{y} k_{a}\left(y_{i e}+d \sin \theta_{i e}+k_{\theta} \theta_{i e}\right)\right)+\frac{k_{b}}{k_{a}} \sin \theta_{i e}
\end{array},\right.
$$

where, $k_{x}, k_{y}, k_{\theta}, k_{a}$ and $k_{b}$ are the positive real number, and $k_{a}+k_{b}=1$.

\subsection{Convergence Proof}

In the field of automatic control, the Lyapunov stability can be used to describe the stability of the dynamic system. According to Sections 3.1-3.3, the Lyapunov function selected in this article is shown in Equation (29). Then

$$
\begin{aligned}
V\left(x_{i e}, y_{i e}, \theta_{i e}\right)= & \left(1+e^{-t}\right) \times \frac{1}{2}\left(x_{i e}-d\left(1-\cos \theta_{i e}\right)\right)^{2}+\left(1+e^{-t}\right) \times \frac{1}{2}\left(y_{i e}-d \sin \theta_{i e}+k_{\theta} \theta_{i e}\right)^{2} \\
& +\left(1+e^{-t}\right) \times \frac{1}{k_{y}}\left(1-\cos \theta_{i e}\right)
\end{aligned}
$$


Obviously, $\left(1+e^{-t}\right)\left(x_{i e}-d\left(1-\cos \theta_{i e}\right)\right)^{2} / 2$ and $\left(1+e^{-t}\right)\left(y_{i e}-d \sin \theta_{i e}+k_{\theta} \theta_{i e}\right)^{2} / 2$ are greater than or equal to 0 respectively. $\left(1+e^{-t}\right)\left(1-\cos \theta_{i e}\right) / k_{y}$ is also greater than or equal to 0 . So, the function $V\left(x_{i e}, y_{i e}, \theta_{i e}, t\right)$ positives definite.

According to Equation (29), for function $V\left(x_{i e}, y_{i e}, \theta_{i e}, t\right)$ after derivation, the following Equation (30) is obtained.

Then

$$
\begin{aligned}
\dot{V}\left(x_{i e}, y_{i e}, \theta_{i e}\right) & =\left(1+e^{-t}\right) \times \frac{1}{2}\left(x_{i e}-d\left(1-\cos \theta_{i e}\right)\right) \times\left(\dot{x}_{i e}-d \sin \theta_{i e} \dot{\theta}_{i e}\right) \\
& +\left(1+e^{-t}\right) \times \frac{1}{2}\left(y_{i e}+d \sin \theta_{i e}+k_{\theta} \theta_{i e}\right) \times\left(\dot{y}_{i e}+d \cos \theta_{i e} \dot{\theta}_{i e}+k_{\theta} \dot{\theta}_{i e}\right) \\
& +\left(1+e^{-t}\right) \times \frac{1}{k_{y}} \sin \theta_{i e} \dot{\theta}_{i e}-e^{-t} \times \frac{1}{2}\left(x_{i e}-d\left(1-\cos \theta_{i e}\right)\right)^{2} \\
& +e^{-t} \times \frac{1}{2}\left(y_{i e}-d \sin \theta_{i e}+k_{\theta} \theta_{i e}\right)^{2}+e^{-t} \times \frac{1}{k_{y}}\left(1-\cos \theta_{i e}\right)
\end{aligned}
$$

By introducing $\left(k_{a}+k_{b}=1\right)$ and Equation (26) into Equation (30), Equation (31) can be obtained. Then

$$
\begin{aligned}
\dot{V}\left(x_{i e}, y_{i e}, \theta_{i e}\right) & =\left(1+e^{-t}\right)\left\{-k_{x}\left(x_{i e}-d\left(1-\cos \theta_{i e}\right)\right)^{2}-v_{v} k_{y} k_{\theta} k_{a}\left(y_{i e}+d \sin \theta_{i e}+k_{\theta} \theta_{i e}\right)^{2}-\frac{k_{b} v_{v}}{k_{y} k_{\theta}} \sin \theta\right\} \\
& -e^{-t}\left\{\frac{1}{2}\left(x_{i e}-d\left(1-\cos \theta_{i e}\right)\right)^{2}+\frac{1}{2}\left(y_{i e}-d \sin \theta_{i e}+k_{\theta} \theta_{i e}\right)^{2}+\frac{1}{k_{y}}\left(1-\cos \theta_{i e}\right)\right\} \leq 0
\end{aligned}
$$

The function $\dot{V}\left(x_{i e}, y_{i e}, \theta_{i e}, t\right)$ is known from Equation (31). It negatives definite.

Lyapunov stability main criterion is often used to prove the stability of the system. Let the state equation of the system be: $\dot{x}=f(x)$, if there is a scalar function $V(x)$ with continuous first derivative and it satisfies that $V(x)$ is positive definite and $\dot{V}(x)$ is negative definite. Then, the system is asymptotically stable in the equilibrium state [20].

According to Lyapunov stability main criterion, the function $V\left(x_{i e}, y_{i e}, \theta_{i e}, t\right)$ positive definite, and $\dot{V}\left(x_{i e}, y_{i e}, \theta_{i e}, t\right)$ is negative definite, so the above control law makes the error system in the equilibrium state asymptotic stability.

\section{Simulation Experiment by MATLAB}

\subsection{Experimental Condition}

This article sets the distance between the wheel axis center and the geometric center of the robot to $0.01 \mathrm{~m}$, that is, $d=0.01 \mathrm{~m}$. It is specified that the maximum linear speed and maximum angular speed of the leader robot are $1 \mathrm{~m} / \mathrm{s}$ and $0.2 \mathrm{rad} / \mathrm{s}$, respectively. The initial coordinate position of the leader robot is set at the coordinate origin under the local coordinate system.

\subsection{Multi-Formation Simulation}

The research objects are one navigation robot and four following robots. The angle between the motion direction of the navigation robot and the positive direction of the $x$-axis is $\theta$. Its initial is 0 . The initial coordinates are $(0,0)$. The initial coordinates of the other six following robots are set as $(-3,-3),(2,4),(8,-4),(-4,2)$ respectively. The simulation results are shown in Figure 8. The $x$-axis and $y$-axis are the coordinate changes during the motion of each robot in Figure 8a. The orange solid line is the desired circular trajectory of the leader robot. The other pink, black, green and blue solid lines are the four motion trajectories following the robot. The horizontal axis and vertical axis respectively reflect the coordinate changes of each robot in the motion process. While maintaining the formation, the robot tracks the leader robot to make a circular motion. The curves of formation position error over time are shown in Figure 8b. The horizontal axis represents time. The vertical axis is the formation position error. 


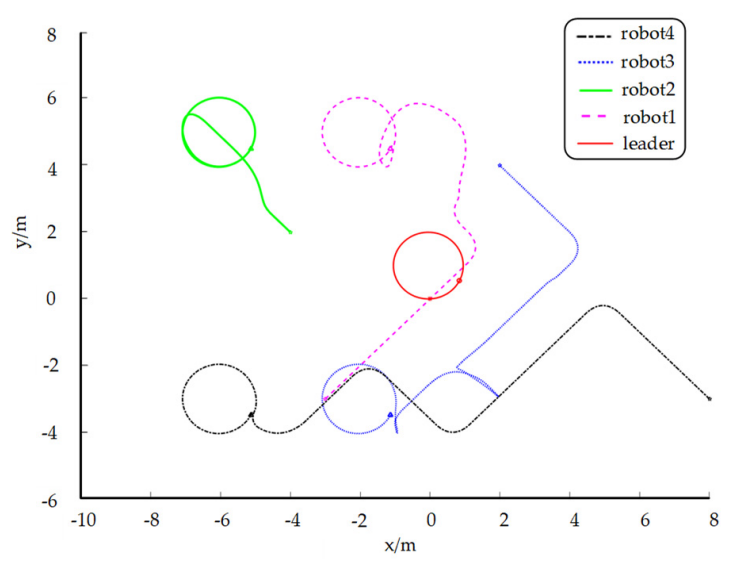

(a)

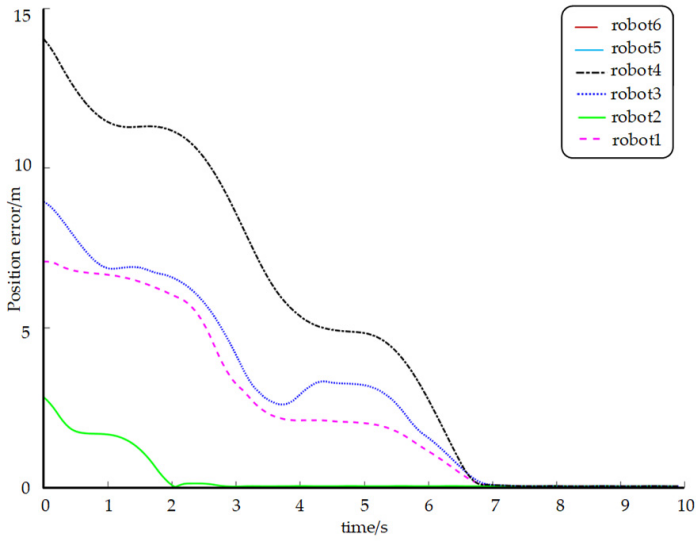

(b)

Figure 8. (a) Formation trajectory of each follower robot and leader robot; (b) Formation position error of each following robot and leader robot.

The influence of algorithm control parameters on system convergence is discussed below. According to Equation (24), it is assumed that the robot formation position error is set as Equation (32). Then

$$
e^{f}=\left\{\begin{array}{ll}
\sqrt{\left(x_{i e}^{2}+y_{i e}^{2}\right)} & x_{i e}>0 \\
-\sqrt{\left(x_{i e}^{2}+y_{i e}^{2}\right)} & x_{i e}<0
\end{array} .\right.
$$

In Figure 8, Figure 8a,b describe the formation of each follower robot and the leader robot in the multi-robot system into two combined formations of triangle and column when the leader makes a circular motion. When $t=0 \mathrm{~s}$, the error is large. As the robot gradually forms a formation, the error gradually decreases. Finally, when the leader robot forms a formation with four following robots, the error becomes zero, and the four following robots track the motion trajectory of the leader robot.

The convergence time refers to the time required for the multiple robots to form the cooperative formation. The smaller the convergence time, the better the algorithm. The position error variation curves are shown in Figure $8 \mathrm{~b}$. In Figure $8 \mathrm{~b}$, it can be obtained that the formation error converges to zero when $t=7 \mathrm{~s}$. To verify the applicability of the algorithm in this article for different numbers of odd and even robots in a multi-robot system, the six robots are employed as an example to form a formation, as shown in Figures 9 and 10.

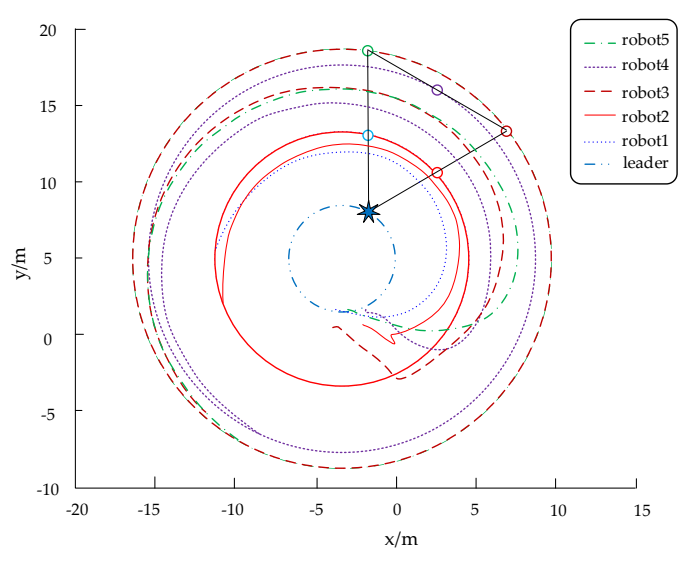

(a)

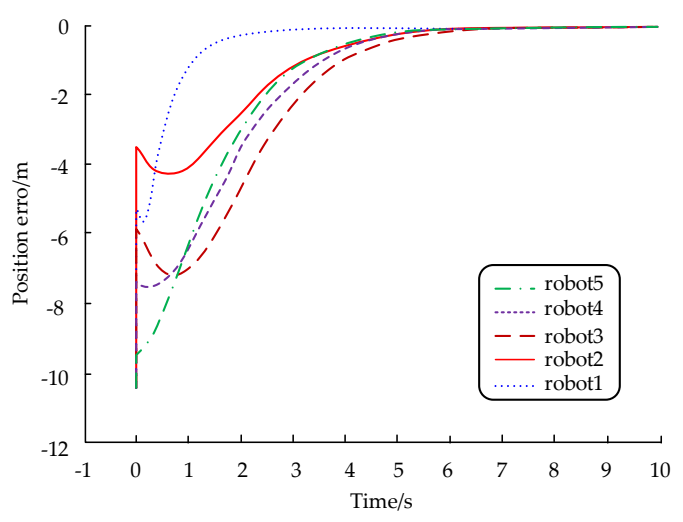

(b)

Figure 9. (a) Trajectory of triangular formation by GCF; (b) Position error of triangular formation. 


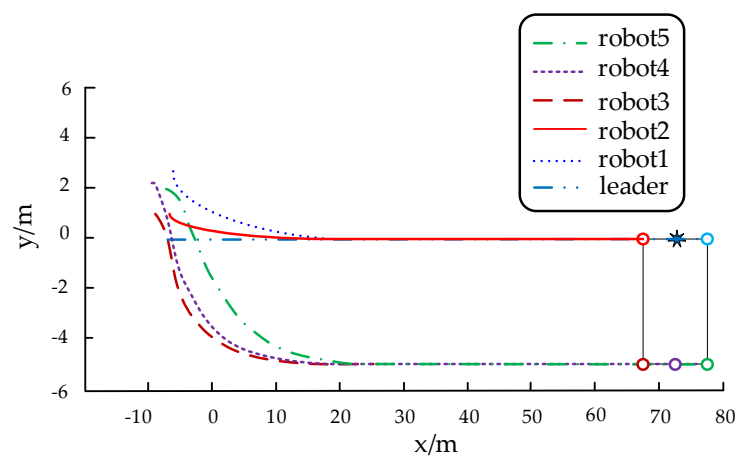

(a)

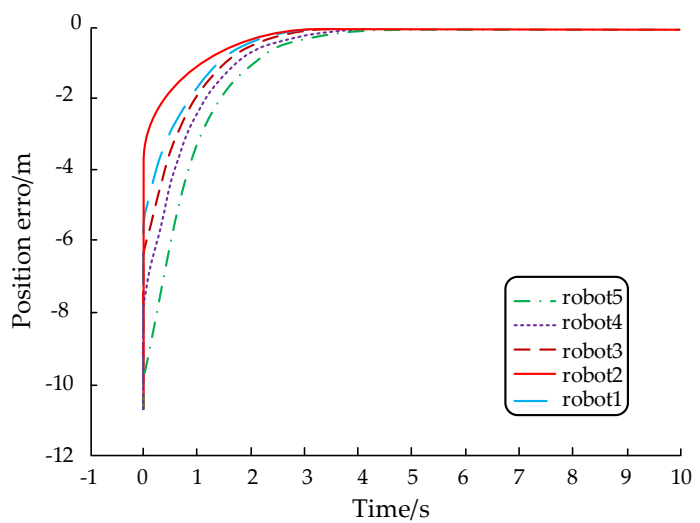

(b)

Figure 10. (a) Trajectory of rectangular formation by GCF; (b) Position error of rectangular formation over time.

The motion trajectories of six robots are shown in Figure 9a, when a triangular formation is formed. The $\mathrm{x}$-axis and $\mathrm{y}$-axis are the coordinate changes during the motion of each robot. The curves of formation position error over time are shown in Figures $9 \mathrm{~b}$ and $10 \mathrm{~b}$. The horizontal axis represents time. The vertical axis is the formation position error. The variation diagram of the position error of a multi-robot system with time is shown in Figure $9 \mathrm{~b}$. The diagram shows that the final multi-robot system would converge when $t=6 \mathrm{~s}$. The position error variation diagram is shown in Figure 10b. Based on Figure 10b, it can be concluded that the final multi-robot system would converge when $t=4 \mathrm{~s}$.

In order to illustrate the effectiveness of the group consistency control designed in this article, the comparison of the two with and without group strategy are performed. The robot trajectory diagram and the system error diagram are obtained by formation control without using the group strategy, as shown in Figure 11. The trajectory diagram and system error diagram of the robot are gained by applying the group strategy, as shown in Figure 12.

The $x$-axis and $y$-axis are the coordinate changes during the motion of each robot in Figures 11a and 12a. The curves of formation position error over time are shown in Figures $11 \mathrm{~b}$ and $12 \mathrm{~b}$. The horizontal axis represents time. The vertical axis is the formation position error. The comparison can be implemented between Figures $11 \mathrm{~b}$ and $12 \mathrm{~b}$. Then, it can be concluded that the convergence time of the formation control using the group strategy is less than that of the algorithm without the group strategy under the same formation.

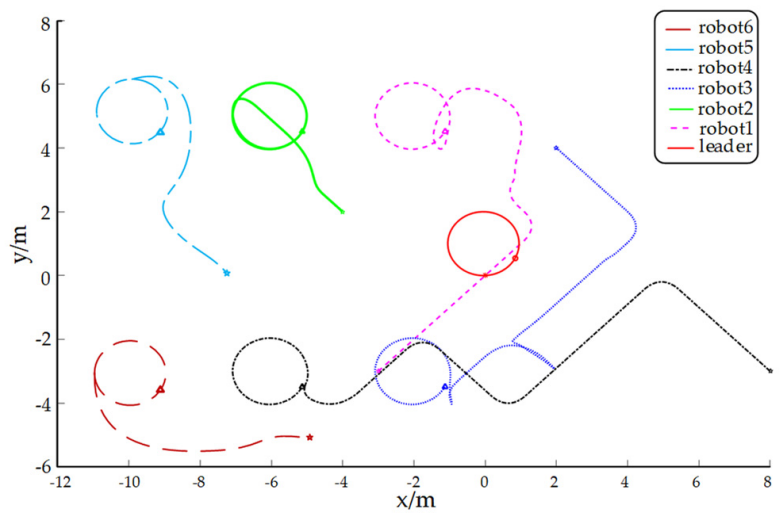

(a)

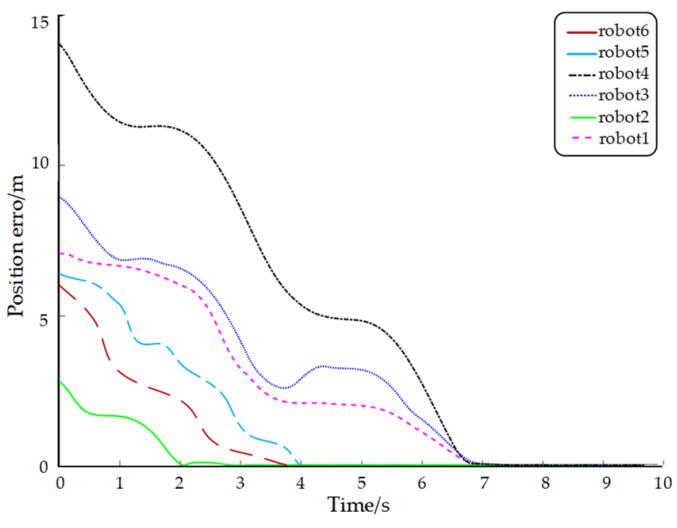

(b)

Figure 11. (a) Trajectories of multi-robot on the plane without grouping strategy; (b) Formation position error of each following robot. 


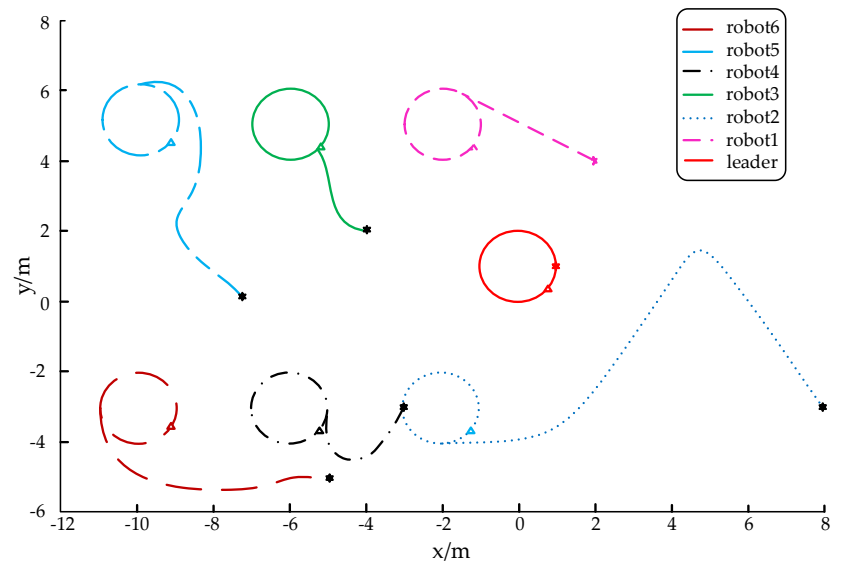

(a)

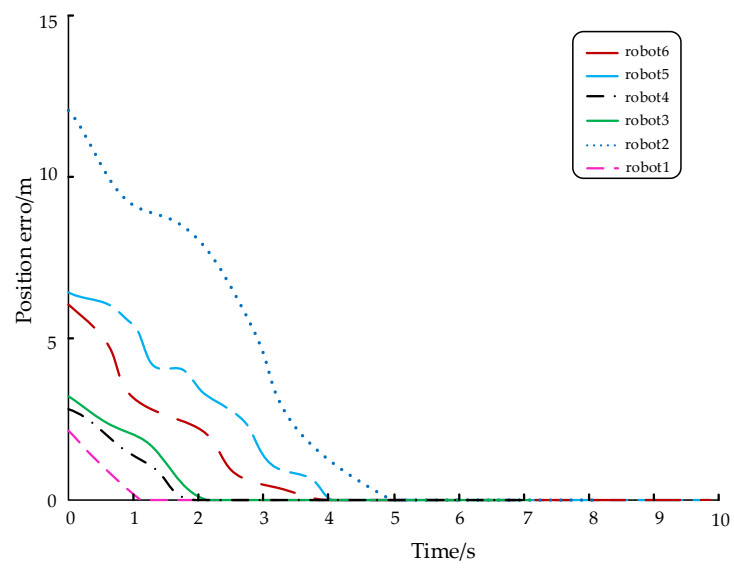

(b)

Figure 12. (a) Trajectories of multi-robot on the plane by GCF; (b) Formation position error of each following robot.

In order to better show the feasibility of the algorithm, the comparison is performed with other algorithms. It takes the spiral formation of the two algorithms, which are the same as the leader-follower formation method, in their respective optimal states. Among them, the practical formation control (PFC) is the method for an anonymous multi-robot system with unknown nonlinear disorders [2]. The trajectory diagram and system error diagram of the robot are obtained by applying PFC, as shown in Figure 13. The trajectory diagram and system error diagram of the robot are acquired by applying GCF, as shown in Figure 14.

The $\mathrm{x}$-axis and $\mathrm{y}$-axis are the coordinate changes during the motion of each robot in Figures 13a and 14a. The curves of formation position error over time are shown in Figures $13 \mathrm{~b}$ and $14 \mathrm{~b}$. The horizontal axis represents time. In Figure $13 \mathrm{~b}, r$ represents the trajectory radius of the robot centered on the point 'target' as the vertical axis. $\alpha$ represents the rotation angle of the robot in motion as the vertical axis. According to Figures 13 and 14, the comparison results are shown in Table 1 for forming spiral formation.

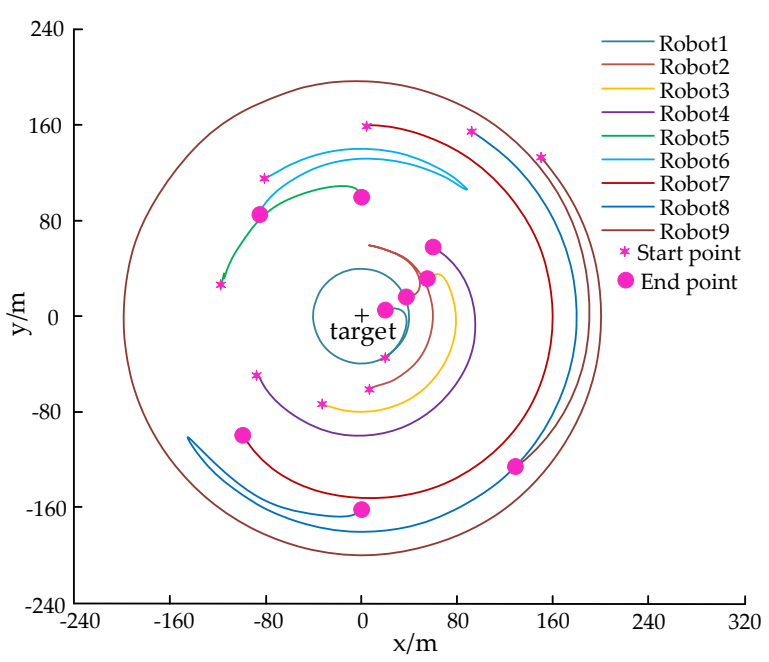

(a)
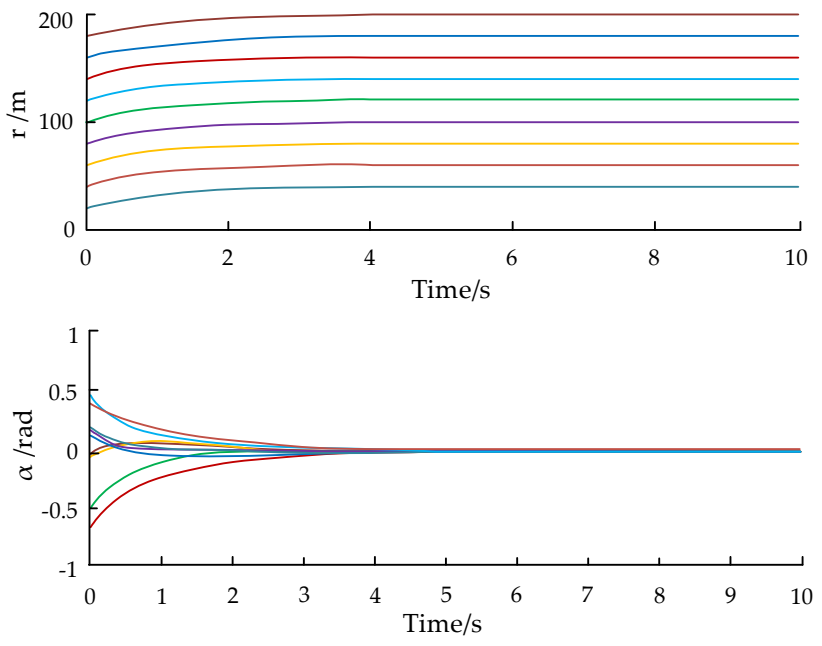

(b)

Figure 13. (a) Trajectories of nine robots on the plane by PFC in spiral formation; (b) Formation position error of each following robot. 


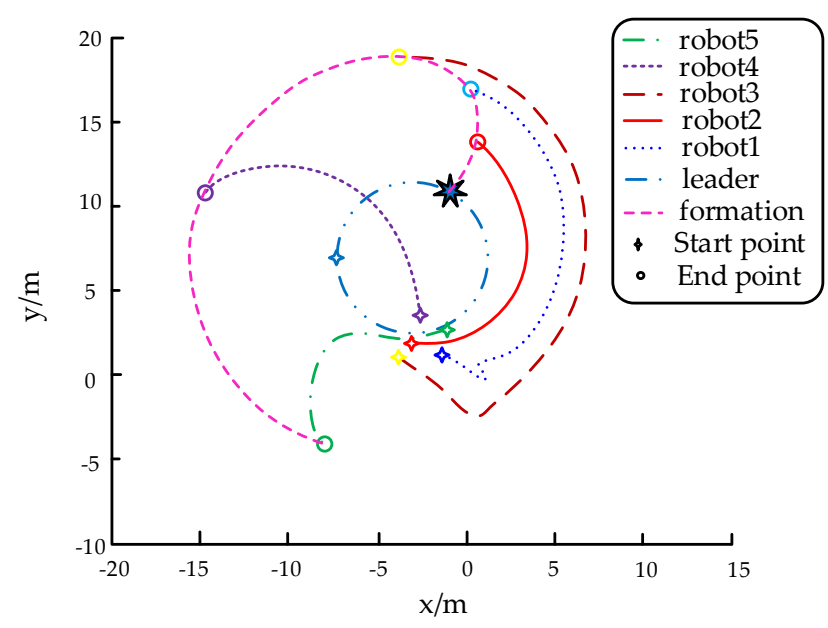

(a)

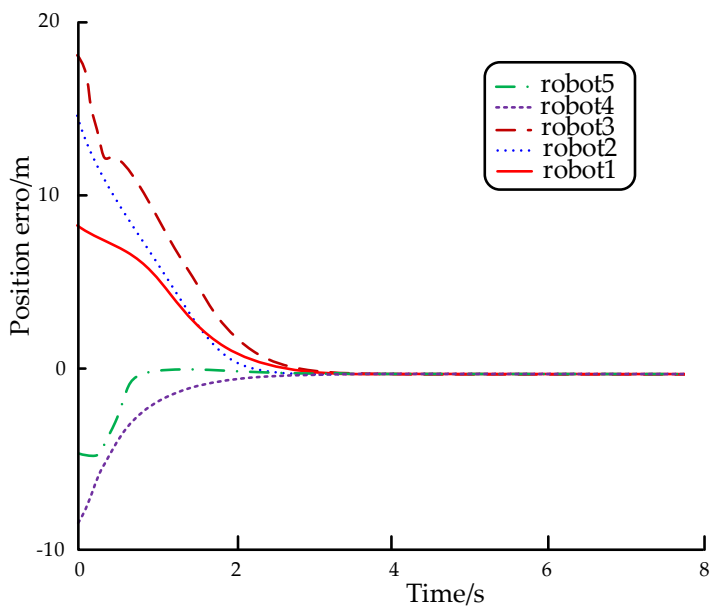

(b)

Figure 14. (a) Trajectories of ten robots on the plane by GCF in spiral formation; (b) Formation position error of each following robot.

Table 1. Data comparison table between PFC and GCF.

\begin{tabular}{ccc}
\hline Project & PFC & GCF \\
\hline$v_{\max }(\mathrm{m} / \mathrm{s})$ & 30 & 30 \\
$l_{F, L}(\mathrm{~m})$ & 40 & 40 \\
Convergence time $(\mathrm{s})$ & 4 & 3 \\
Number of robots & 9 & 6 \\
\hline
\end{tabular}

To sum up, according to Figures 8-10, the simulation results show that the multimobile robots using the algorithm control law in this article can dynamically form formation during operation on the different formation problems. Through the comparison of the two algorithms in Table 1, it can be seen that the convergence time of the algorithm in this article will be $25 \%$ less than that of PFC. Compared with PFC, the number of robots is poor. However, the overall comparison of the formation control algorithm in this article is acceptable in theoretical analysis and simulation. Therefore, the formation control algorithm proposed in this article has effectiveness and feasibility.

\section{Conclusions and Future Work}

Based on the theory of automatic control and the nonholonomic system, this article studies the formation problem of nonholonomic constrained multi-robot tracking the leader's movement while maintaining the ideal formation. The formation communication protocol of multi-robot cooperative movement is established. Through the mathematical analysis of nonholonomic constraints, the motion models and motion system error models of the leader robot and follower robot are constructed respectively. Then the convergence of formation control strategy in this article is proved theoretically by the Lyapunov function. The feasibility of formation control of the multi-robot system with the nonholonomic constraints is verified by MATLAB simulation. By comparing with the simulation results of other navigator formation control algorithms, the effectiveness of the GCF algorithm is verified.

In order to illustrate the effectiveness of the group consistency control designed in this article, the comparison of the two with and without group strategy are implemented. Then, two simulation results are obtained. The comparison of the two simulation results shows that the convergence time of formation control using the group strategy is less than that of the algorithm without the group strategy under the same formation. In order to better show the feasibility of the algorithm proposed, the comparison is performed with PFC 
algorithms. Through the comparison of the two algorithms, the convergence time of the algorithm in this article can be $25 \%$ less than that of PFC. Compared with PFC, the number of robots is poor. However, the overall comparison of the formation control algorithm can be acceptable in theoretical analysis and simulation. Hence, the formation control algorithm has effectiveness and feasibility. Because there are many uncertain conditions in the real robot systems, such as external noise and nonlinear interference, the formation control problem of nonholonomic constrained multi-mobile robot systems with external interference would be studied in the future.

Author Contributions: Conceptualization, H.Z.; methodology, H.Z. and K.D.; formal analysis, N.W. and L.D.; data curation, H.Z. and K.D.; writing-original draft preparation, H.Z. and K.D.; writingreview and editing, H.Z.; visualization, K.D. and N.W.; supervision, H.Z; funding acquisition, H.Z. All authors have read and agreed to the published version of the manuscript.

Funding: This research was funded by the National Natural Science Foundation of China, grant number 51505335; the Industry University Cooperation Collaborative Education Project of the Department of Higher Education of the Ministry of Education of China, grant number 202102517001; the Doctor Startup Project of TUTE, grant number KYQD 1806.

Institutional Review Board Statement: Not applicable.

Informed Consent Statement: Not applicable.

Data Availability Statement: Not applicable.

Conflicts of Interest: The authors declare no conflict of interest.

\section{References}

1. Karras, G.C.; Bechlioulis, C.P.; Fourlas, G.K.; Kyriakopoulos, K.J. A mixed-initiative formation control strategy for multiple quadrotors. Robotics 2021, 10, 116. [CrossRef]

2. Xu, P.; Tao, J.; Xu, M.; Xie, G. Practical formation control for multiple anonymous robots system with unknown nonlinear disturbances. Appl. Sci. 2021, 11, 9170. [CrossRef]

3. Elaamery, B.; Pesavento, M.; Aldovini, T.; Lissandrini, N.; Michieletto, G.; Cenedese, A. Model predictive control for cooperative transportation with feasibility-aware policy. Robotics 2021, 10, 84. [CrossRef]

4. Rashid, M.; Yakub, F.; Zaki, S.; Ali, M.; Mamat, N.; Putra, S.; Roslan, S.; Shah, H.; Aras, M. Comprehensive review on controller for leader-follower robotic system. Indian J. Geo-Mar. Sci. 2019, 48, 985-1007.

5. Draganjac, I.; Miklić, D.; Kovačić, Z.; Vasiljević, G.; Bogdan, S. Decentralized control of multi-AGV systems in autonomous warehousing applications. IEEE Trans. Autom. Sci. Eng. 2016, 13, 1433-1447. [CrossRef]

6. Zhu, H.; Claramunt, F.M.; Brito, B.; Alonso-Mora, J. Learning interaction-aware trajectory predictions for decentralized multi-robot motion planning in dynamic environments. IEEE Robot. Autom. Lett. 2021, 6, 2256-2263. [CrossRef]

7. Luis, C.E.; Vukosavljev, M.; Schoellig, A.P. Online trajectory generation with distributed model predictive control for multi-robot motion planning. IEEE Robot. Autom. Lett. 2020, 5, 604-611. [CrossRef]

8. Défago, X.; Souissi, S. Non-uniform circle formation algorithm for oblivious mobile robots with convergence toward uniformity. Theor. Comput. Sci. 2008, 396, 97-112. [CrossRef]

9. Flocchini, P.; Prencipe, G.; Santoro, N. Self-deployment of mobile sensors on a ring. Theor. Comput. Sci. 2008, 402, 67-80. [CrossRef]

10. Zhuang, H.C.; Xia, Y.L.; Wang, N.; Dong, L. High inclusiveness and accuracy motion blur real-time gesture recognition based on YOLOv4 model combined attention mechanism and DeblurGanv2. Appl. Sci. 2021, 11, 9982. [CrossRef]

11. Zhuang, H.C.; Dong, K.L.; Qi, Y.M.; Wang, N.; Dong, L. Multi-destination path planning method research of mobile robots based on goal of passing through the fewest obstacles. Appl. Sci. 2021, 11, 7378. [CrossRef]

12. Brinón-Arranz, L.; Seuret, A.; Canudas-de-Wit, C. Cooperative control design for time-varying formations of multi-robot systems. IEEE Trans. Autom. Control 2014, 59, 2283-2288. [CrossRef]

13. Zheng, R.; Liu, Y.; Sun, D. Enclosing a target by nonholonomic mobile robots with bearing-only measurements. Automatica 2015, 53, 400-407. [CrossRef]

14. Yu, X.; Liu, L. Cooperative control for moving-target circular formation of nonholonomic vehicles. IEEE Trans. Autom. Control 2016, 62, 3448-3454. [CrossRef]

15. Wang, C.; Xie, G.; Cao, M. Controlling anonymous mobile agents with unidirectional locomotion to form formations on a circle. Automatica 2014, 50, 1100-1108. [CrossRef]

16. Peng, L.; Ren, W.; Song, Y.D. Distributed multi-robot optimization subject to nonidentical constraints and communication delays. Automatica 2016, 65, 120-131.

17. Zhuang, H.C.; Wang, N.; Gao, H.B.; Deng, Z.Q. Quickly obtaining range of articulated rotating speed for electrically driven large load-ratio six-legged robot based on maximum walking speed method. IEEE Access 2019, 7, 29453-29470. [CrossRef] 
18. Wang, C.; Xie, G. Limit-cycle-based decoupled design of circle formation control with collision avoidance for anonymous agents in a plane. IEEE Trans. Autom. Control 2017, 62, 6560-6567. [CrossRef]

19. Zhuang, H.C.; Wang, N.; Gao, H.B.; Deng, Z.Q. Autonomous fault-tolerant gait planning research for electrically driven largeload-ratio six-legged robot. In Proceedings of the 12th International Conference on Intelligent Robotics and Applications (ICIRA 2019), Shenyang, China, 8-11 August 2019; pp. 231-244.

20. Wang, C.; Xie, G.; Cao, M. Forming circle formations of anonymous mobile robots with order preservation. IEEE Trans. Autom. Control 2013, 58, 3248-3254. [CrossRef]

21. Liu, Z.; Zhuang, H.C.; Gao, H.B.; Deng, Z.Q.; Ding, L. Static force analysis of foot of electrically driven heavy-duty six-legged robot under tripod gait. Chin. J. Mech. Eng. 2018, 31, 1-15. [CrossRef]

22. Zhuang, H.C.; Gao, H.B.; Deng, Z.Q. Gait planning research for an electrically driven large-load-ratio six-legged robot. Appl. Sci. 2017, 7, 296. [CrossRef]

23. Liu, D.; Zong, C.; Wang, D.; Zhao, W.; Wang, Y.; Lu, W. Multi-robot formation control based on high-order bilateral consensus. Meas. Control 2020, 53, 983-993. [CrossRef]

24. Liu, R.; Cao, X.; Liu, M. Finite-time synchronization control of spacecraft formation with network-induced communication delay. IEEE Access 2017, 5, 27242-27253. [CrossRef]

25. Song, C.; Liu, L.; Feng, G.; Xu, S.Y. Coverage control for heterogeneous mobile sensor networks on a circle. Automatica 2016, 63, 349-358. [CrossRef]

26. Chen, Y.T.; Chiu, C.S.; Lee, Y.T. Grey estimator-based tracking controller applied to swarm robot formation. Axioms 2021, 10, 298. [CrossRef] 